\title{
Redução da primeira dose de GnRH em vacas holandesas de alta produção sincronizadas com Ovsynch ou Heatsynch
}

\author{
Reduction of first GnRH dosage in high-yielding holstein cows synchronized with Ovsynch \\ or Heatsynch
}

\author{
Flávio Aragon LIMA ${ }^{1}$; Márcio Barciela VERAS ${ }^{1}$; José Nélio de Sousa SALES ${ }^{3}$; Gabriel Armond \\ CREPALDI ${ }^{3}$; José Ricardo Garla de MAIO $^{2}$; Pietro Sampaio BARUSELLI ${ }^{3}$ \\ ${ }^{1}$ Policlínica Veterinária Pioneiros, Carambei-PR \\ ${ }^{2}$ Ouro Fino Saúde Animal, Ribeirão Preto-SP \\ ${ }^{3}$ Departamento de Reprodução Animal da Faculdade de Medicina Veterinária e Zootecnia da Universidade de São Paulo, São Paulo-SP
}

\begin{abstract}
Resumo
O objetivo desse estudo foi avaliar o efeito da redução da primeira dose de GnRH (10 vs $20 \mu \mathrm{g}$ ) e do tipo de protocolo de sincronização para a IATF (Ovsynch e Heatsynch) na taxa de prenhez de vacas holandesas de alta produção sincronizadas para IATF $(\mathrm{n}=581)$. Os animais foram distribuídos entre os tratamentos e alocados em arranjo fatorial $2 \times 2$, sendo: Trat 1- Ovsynch $(20 \mu \mathrm{g}$ GnRH no D0; PGF no D7; $10 \mu \mathrm{g}$ GnRH no D9 e IATF no D10; n = 141), Trat 2- 1/2 Ovsynch (10 $\mu \mathrm{g}$ GnRH no D0; PGF no D7; $10 \mu g$ GnRH no D9 e IATF no D10; n = 159), Trat 3- Heatsynch (20 $\mu \mathrm{g}$ GnRH no D0; PGF no D7; 1 mg BE no D8 e IATF no D10; $n=147)$ e Trat 4- 1/2 Heatsynch (10 $\mu$ g GnRH no D0; PGF no D7; 1 mg BE no D8 e IATF no D10; $\mathrm{n}=134$ ). O Grupo Ovsynch (Trat 1 e 2) apresentou maior taxa de prenhez que o grupo Heatsynch (Trat $3 \mathrm{e}$ 4) aos 28 dias (45,7\% (137/300) e 39,9\% (112/281), respectivamente; $P=0,07)$ e aos 56 dias de gestação $(41,0 \%(123 / 300)$ e 34,2\% (96/281), respectivamente; $P=0,06)$. A dose de $\mathrm{GnRH}$, não influenciou $(P>0.10)$ a taxa de prenhez aos 28 dias $(44,4 \%(128 / 288)$ vs $41,3 \%(121 / 293) ; P=0,50)$ e aos 56 dias $(38,2 \%(110 / 288)$ vs $37,2 \%(109 / 293) ; P=0,68)$ entre os grupos tratados com $10 \mu \mathrm{g}$ ou $20 \mu \mathrm{g}$ de acetato de buserelina. As perdas gestacionais (ente 28 e 56 dias) não diferiram entre os protocolos para IATF e dose de GnRH $(P>0,10)$. Conclui-se que, protocolo Ovsynch apresentou maior taxa de prenhez à IATF que o Heatsynch e a administração de $10 \mu \mathrm{g}$ de Acetato de Buserelina no início dos tratamentos de sincronização não comprometeu a eficiência do protocolo de sincronização para IATF em vacas holandesas de alta produção.
\end{abstract}

Palavras-chave: IATF. Sincronização da ovulação. Dose GnRH. Vacas de leite.

\begin{abstract}
The objective of this study was to evaluate the effect of treatment with 10 or $20 \mu \mathrm{g}$ of GnRH and type of FTAI protocols (Ovsynch and Heatsynch) on pregnancy rate of lactating dairy cows submitted to the synchronization at FTAI $(\mathrm{n}=581)$. The animals were distributed among four treatments, allocated factorial design $2 \times 2$ : Treat 1- Ovsynch $(20 \mu \mathrm{g} \mathrm{GnRH}$ on D0; PGF on D7; $10 \mu \mathrm{g}$ GnRH on D9 and FTAI on D10; $\mathrm{n}=141$ ), Treat 2- 1/2 Ovsynch (10 $\mu \mathrm{g} \mathrm{GnRH}$ on D0; PGF on D7; $10 \mu \mathrm{g}$ GnRH on D9 and FTAI on D10; $\mathrm{n}=159)$, Treat 3- Heatsynch $(20 \mu \mathrm{g}$ GnRH on D0; PGF on D7; $1 \mathrm{mg}$ EB on D8 and FTAI on D10; $\mathrm{n}=147)$ and Treat 4- $1 / 2$ Heatsynch $(10 \mu \mathrm{g}$ GnRH on D0; PGF on D7; $1 \mathrm{mg}$ EB on D8 and FTAI on $\mathrm{D} 10 ; \mathrm{n}=134$ ). The Group Ovsynch (Treat 1 and 2) presented greater pregnancy rate than Group Heatsynch (Treat 3 and $4)$ at 28 days $(45.7 \%(137 / 300)$ and $39.9 \%(112 / 281)$, respectively; $P=0.07)$ and at 56 days of gestation $(41.0 \%(123 / 300)$ and $34.2 \%(96 / 281)$, respectively; $P=0.06)$. There were no statistical difference $(P>0.10)$ on pregnancy rate at 28 (44.4\% (128/288) vs $41.3 \%(121 / 293) ; P=0.50)$ and 56 days $(38.2 \%(110 / 288)$ vs $37.2 \%(109 / 293) ; P=0.68)$ between the dosages of the GnRH. The pregnancies losses (between 28 and 56 days) were not different between the FTAI protocols and GnRH dosage $(\mathrm{P}>0.10)$. We concluded that the Ovsynch protocol presented greater pregnancy rate at FTAI than Heatsynch protocol and the administration of $10 \mu \mathrm{g}$ of buserelin Acetate, at the beginning of these protocols did not decrease the efficiency of FTAI protocols in high-yielding Holstein cows.
\end{abstract}

Keywords: FTAI. Ovulation synchronized. Dosage GnRH. Dairy cows.

\section{Introdução}

Em todo o mundo há relatos que indicam baixa taxa de serviço em bovinos inseminados artificialmente, principalmente em decorrência de falhas na detecção do cio. Quando poucas vacas são detectadas em cio ocorrem perdas significativas na eficiência reprodutiva
Correspondência para:

Pietro Sampaio Baruselli

Departamento de Reprodução Animal, FMVZ/USP, Avenida Professor Dr. Orlando Marques de Paiva, 87. Cidade Universitária CEP 05508-270, São Paulo-SP, Brasil. e-mail: znlogan@yahoo.com.br, barusell@usp.br

Recebido para publicação: 28/05//2008

Aprovado para publicação: 29/10/2009 
do rebanho e comprometimento do programa de inseminação $\operatorname{artificial}^{1}$. Com o intuito de acabar com os efeitos da baixa detecção de cio na eficiência reprodutiva, Thatcher et al. $^{2}$ e Pursley, Mee e Wiltbank ${ }^{3}$ desenvolveram métodos de sincronização da ovulação para permitir a inseminação artificial em tempo predeterminado, que consistem na emergência sincronizada de uma onda de crescimento folicular, regressão do corpo lúteo e ovulação do folículo dominante com hormônio liberador de gonadotrofina $(\mathrm{GnRH})$ e prostaglandina (PGF). A sincronização da ovulação para inseminação artificial em tempo fixo aumenta o número de vacas inseminadas após o período voluntário de espera e, consequentemente, aumenta a taxa de prenhe $\mathrm{z}^{4,5}$.

Em rebanhos de leite, Pursley, Mee e Wiltbank ${ }^{3}$ propuseram um método de sincronização, que consiste em uma primeira administração intramuscular de GnRH, sem levar em conta o período do ciclo estral. O tratamento com GnRH induz a ovulação do folículo dominante, quando presente no momento do tratamento, promovendo o início sincronizado de uma nova onda de crescimento folicular. Após sete dias é realizado o tratamento com prostaglandina com a finalidade de regredir o corpo lúteo presente. Se esse for decorrente do tratamento inicial de $\mathrm{GnRH}$, ocorrerá tempo suficiente para responder ao agente luteolítico. Uma segunda dose de GnRH é administrada $48 \mathrm{~h}$ após a PGF, com a finalidade de promover a ovulação sincronizada do folículo dominante. A inseminação artificial é realizada 16 à $18 \mathrm{~h}$ após o último tratamento hormonal, sem a necessidade de detecção do estro. Esse protocolo ficou conhecido como "Ovsynch". Posteriormente, estudos foram realizados para tentar substituir o último GnRH do protocolo Ovsynch por estradiol ${ }^{6,7}$. O protocolo com a utilização do estradiol como indutor da ovulação em substituição do último GnRH, ficou conhecido como "Heatsynch".

Inúmeras variantes do protocolo "Ovsynch" foram propostas para tentar reduzir o custo do protocolo de sincronização, dentre elas, a redução da dose de GnRH em $50 \%$ da recomendada, essa redução não afetou as taxas de sincronização $(84,9 \%$ vs $83,1 \%)$ e concepção ( $41,0 \%$ vs $41,1 \%$ ) entre os grupos que receberam doses reduzida ou cheia, respectivamente ${ }^{8}$. Assim, a diminuição da dose de GnRH acarretou redução de custos sem comprometer a eficácia do programa de sincronização. Resultados similares foram encontrados por Oliveira e Ribeiro Filho ${ }^{9}$ em vacas da raça Zebu, os quais empregaram também metade da dose de GnRH. Outra forma de reduzir o custo foi a utilização de éster de estradiol como indutor da ovulação, em substituição ao último GnRH do protocolo Ovsynch sem alteração significativa nas taxas de prenhez ${ }^{6,7,10}$.

O objetivo desse experimento foi avaliar o efeito da redução da primeira dose de GnRH e do tipo de protocolo de sincronização para inseminação artificial em tempo fixo (Ovsynch ou Heatsynch) na taxa de prenhez de vacas holandesas de alta produção.

\section{Material e Método}

O experimento foi realizado em cinco fazendas localizadas na região de Castro, no estado do Paraná, e conduzido de agosto a setembro de 2006, sendo os tratamentos realizados simultaneamente. Foram utilizadas 581 vacas holandesas em lactação $(144,1 \pm 5,2$ dias de lactação) com produção média de 37,1 \pm 9,4 $\mathrm{kg} /$ dia de leite e escore de condição corporal 2,80 \pm $0,31$ (escala de 1 a 5$)^{11}$. Os animais do experimento eram ordenhados duas vezes ao dia, mantidos em sistema de free stall e alimentados duas vezes ao dia com dieta a base de silagem de milho e concentrado (milho moído, farelo de soja, caroço de algodão, fosfato bicálcio, cloreto de sódio, vitaminas e minerais) que atendia as exigências nutricionais de mantença e produção ${ }^{12}$. Para entrar no experimento, os animais foram avaliados por ultrassonografia e aqueles com anormalidades reprodutivas (metrites, endometrites, aderências ovarianas ou uterinas) não foram incluídos no estudo. 
No dia (D0), todos os animais foram classificados, por exame ultrassonográfico, de acordo com a presença ou não de corpos lúteos e a presença de cistos ovarianos (folículos maiores que $20 \mathrm{~mm}$ e sem a presença de corpo lúteo) para dividir os animais de forma equilibrada entre os grupos de tratamentos. Após essa avaliação, os animais foram distribuídos inteiramente ao acaso em quatro tratamentos. Os tratamentos estavam em arranjo fatorial $2 \times 2$ [dois protocolos (Ovsynch vs Heatsynch) e duas doses de GnRH (10 $\mu \mathrm{g}$ vs $20 \mu \mathrm{g}$ de Acetato de Buserelina)] e estão descritos a seguir, seguidos pelos números de animais utilizados em cada tratamento:

- Tratamento 1 - Ovsynch $\rightarrow$ No D0, as vacas receberam $20 \mu \mathrm{g}$ Acetato de Buserelina. Sete dias depois (D7) administrou-se i.m. $500 \mu \mathrm{g}$ de Cloprostenol e no dia 9 (D9), $10 \mu$ g Acetato de Buserelina ( $\mathrm{n}=141$ );

- Tratamento 2 - 1/2 Ovsynch $\rightarrow$ No D0, as vacas receberam $10 \mu \mathrm{g}$ Acetato de Buserelina. O restante do protocolo foi semelhante ao Tratamento 1-Ovsynch $(\mathrm{n}=159)$;

- Tratamento 3 - Heatsynch $\rightarrow$ No D0, as vacas receberam $20 \mu \mathrm{g}$ Acetato de Buserelina. Sete dias de- pois (D7) administrou-se i.m. $500 \mu \mathrm{g}$ de Cloprostenol e no dia 8 (D8), 1 mg de Benzoato de estradiol ( $\mathrm{n}=147)$;

- Tratamento 4 - 1/2 Heatsynch $\rightarrow$ No D0, as vacas receberam $10 \mu \mathrm{g}$ Acetato de Buserelina. O restante do protocolo foi semelhante ao Tratamento 3 -Heatsynch $(\mathrm{n}=134)$.

Os animais dos tratamentos 1 e 2 (Ovsynch) foram inseminados 16 horas após a última administração de GnRH. As vacas dos tratamentos 3 e 4 (Heatsynch) foram inseminadas 40 horas após o tratamento com Benzoato de estradiol. Exames ultrassonográficos foram realizados para verificar as taxas de prenhez aos 28 e 56 dias após a inseminação artificial. As perdas gestacionais foram determinadas pela diferença na taxa de prenhez entre 28 e 56 dias de gestação. Os produtos utilizados para a sincronização foram: Acetato de Buserelina (SincroForte ${ }^{\circledR}$, Ouro Fino, Ribeirão Preto, Brasil), Cloprostenol sódico (Sincrocio ${ }^{\oplus}$, Ouro Fino, Ribeirão Preto, Brasil) e Benzoato de estradiol (Sincrodiol ${ }^{\circledR}$, Ouro Fino, Ribeirão Preto, Brasil). Os protocolos de sincronização estão apresentados na figura 1.

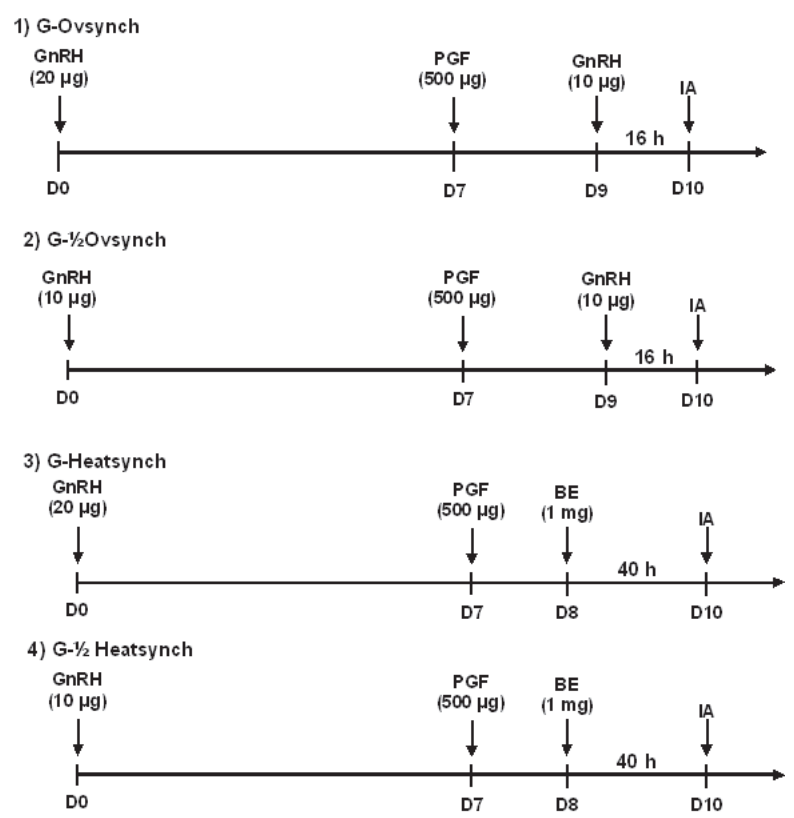

Figura 1 - Diagrama esquemático dos tratamentos 
As análises das taxas de prenhez aos 28 e 56 dias após a inseminação artificial e as perdas gestacionais foram realizadas com auxílio do software Statistical Analysis System for Windows SAS ${ }^{\oplus 13}$. Os efeitos de tipo de protocolo (Ovsynch e Heatsynch) e dose de GnRH foram analisados por regressão logística. O modelo foi construído por um processo de eliminação dos efeitos não significativos, mantendo sempre no modelo os efeitos de dose de GnRH e tipo de protocolo. As variáveis explanatórias utilizadas no modelo foram dias em lactação, condição corporal, número de partos, fazenda e produção de leite, sendo excluídas aquelas com valor de $P>0,1$. As variáveis respostas do experimento foram taxas de prenhez aos 28 e 56 dias e as perdas gestacionais durante esse período. A comparação da taxa de prenhez entre os tratamentos, bem como possíveis interações foram realizadas pelo procedimento GLIMMIX do SAS. Para cada variável resposta foi verificado efeito das variáveis independentes e suas respectivas interações. O nível de significância para rejeitar H0 (hipótese de nulidade) foi de $10 \%$.

\section{Resultados}

Das 581 vacas do experimento, 319 vacas apresentavam corpo lúteo. Ainda, 43 vacas apresentavam cisto folicular. As taxa de prenhez e de perdas gestacionais não diferiram em função da presença ou não de corpo lúteo ou de cisto folicular no início do protocolo experimental (Tabela 1).

Não houve interação $(P>0,10)$ entre as variáveis independentes e explanatórias e entre os protocolos de sincronização e as doses de $\mathrm{GnRH}$ administradas na primeiro dia do protocolo de sincronização. O protocolo Ovsynch (Tratamentos 1 e 2) apresentou maior taxa de prenhez do que o Heatsynch (Tratamentos $3 \mathrm{e}$ 4) aos 28 e 56 dias de gestação ( $P=0,07$ e $P=0,06$; respectivamente, tabela 2). Quanto à dose de GnRH, não se verificou diferença estatística entre os tratamentos GnRH (Tratamentos 1 e 3) e $1 / 2$ GnRH (Tratamentos 2 e 4) nas taxas de prenhez aos 28 e 56 dias $(P=0,50$ e $P=0,68$; respectivamente, Tabela 3 ). As perdas gestacionais não diferiram entre os grupos Ovsynch vs Heatsynch $(P=0,49$; Tabela 2$)$ e GnRH vs $1 / 2$ GnRH $(P=0,56$; Tabela 3$)$.

\section{Discussão}

Os resultados desse experimento indicam que as vacas submetidas ao protocolo Ovsynch apresentaram maior taxa de prenhez quando comparadas às vacas que receberam o protocolo Heatsynch. Resultados semelhantes foram encontrados por Kasimanickam, Cornwell e $\mathrm{Nebel}^{14}$, os quais substituíram a segunda dose de GnRH por Cipionato de estradiol administrados 24 horas após a PGF2a e obtiverem

Tabela 1 - Taxa de prenhez aos 28 e 56 dias e perdas gestacionais em vacas holandesas de alta produção de acordo com o status ovariano diagnosticado por ultrassonografia no início do protocolo de sincronização para IATF - região de Castro-PR - 2008

\begin{tabular}{ccccc}
\hline & \multicolumn{3}{c}{ Estruturas ovarianas } & \multirow{P}{*}{$\boldsymbol{P}$} \\
\cline { 2 - 4 } & Corpo lúteo & Sem CL & Cisto & \\
\hline Taxa de prenhez aos 28 dias (\%) & $43,9(140 / 319)$ & $41,6(91 / 219)$ & $41,9(18 / 43)$ & 0,76 \\
Taxa de prenhez aos 56 dias (\%) & $38,6(123 / 319)$ & $36,9(81 / 219)$ & $37,2(16 / 43)$ & 0,88 \\
Perdas gestacionais (\%) & $12,1(17 / 140)$ & $10,9(10 / 91)$ & $11,1(2 / 18)$ & 0,79 \\
\hline
\end{tabular}


Tabela 2 - Efeito do protocolo de sincronização (Ovsynch vs Heatsynch) na taxa de prenhez aos 28 e 56 dias e nas perdas gestacionais em vacas holandesas de alta produção - região de Castro-PR - 2008

\begin{tabular}{cccc}
\hline & Ovsynch & Heatsynch & $P$ \\
\hline Taxa de prenhez aos 28 dias (\%) & $45,7(137 / 300)$ & $39,9(112 / 281)$ & 0,07 \\
Taxa de prenhez aos 56 dias (\%) & $41,0(123 / 300)$ & $34,2(96 / 281)$ & 0,06 \\
Perdas gestacionais (\%) & $10,2(14 / 137)$ & $14,3(16 / 112)$ & 0,49 \\
\hline
\end{tabular}

Tabela 3 - Efeito da dose do primeiro GnRH ( $10 \mu \mathrm{g}$ vs $20 \mu \mathrm{g}$ Acetato de Buserelina) na taxa de prenhez aos 28 e 56 dias e nas perdas gestacionais vacas holandesas submetidas à IATF - região de Castro-PR - 2008

\begin{tabular}{cccc}
\hline & GnRH (20 $\mu \mathrm{g})$ & $\mathbf{1} / \mathbf{2}$ GnRH (10 $\boldsymbol{\mu g})$ & $\boldsymbol{P}$ \\
\hline Taxa de prenhez aos 28 dias (\%) & $44,4(128 / 288)$ & $41,3(121 / 293)$ & 0.50 \\
Taxa de prenhez aos 56 dias (\%) & $38,2(110 / 288)$ & $37,2(109 / 293)$ & 0,68 \\
Perdas gestacionais (\%) & $14,1(18 / 128)$ & $9,9(12 / 121)$ & 0,56 \\
\hline
\end{tabular}

menor taxa de prenhez aos 28 e 56 dias de gestação. Uma possível explicação para os resultados encontrados nesse estudo é que vacas submetidas ao protocolo Heatsynch apresentam menor taxa de ovulação, conforme verificado em estudos anteriores. ${ }^{15}$ No entanto, diferente dos nossos resultados, Stevenson e Tiffany (dados não publicados segundo Stevenson, Tiffany e Lucy) ${ }^{15}$ relataram não haver diferença na taxa de prenhez entre os protocolos Ovsynch e Heatsynch $[29,2 \%(64 / 219)$ e $27,5 \%(63 / 229)$, respectivamente]. Essa diferença de resultados se deve provavelmente à época em que os experimentos foram realizados. No presente experimento, as inseminações se concentraram em época do ano com clima mais ameno (primavera), o que permitiu taxas de prenhez ao redor de $40 \%$ e a diferença entre os protocolos Ovsynch e Heatsynch.
Em animais previamente sincronizados com dispositivos de progesterona, o intervalo entre a administração do benzoato de estradiol e a ovulação é, em média, 48 horas $^{16,17,18}$. Entretanto, o intervalo entre o tratamento com GnRH e a ovulação é de 27 a 28 h. ${ }^{15,19}$ Sabe-se ainda que o pico de LH ocorre $20 \mathrm{~h}$ após a administração de $\mathrm{BE}^{20,21}$ e duas horas após o tratamento com GnRH (Acetado de buserelina). Levando em consideração esses períodos, pode-se supor que o intervalo entre o tratamento com PGF e a ovulação é de $76 \mathrm{~h}$ para o protocolo Ovsynch e de $72 \mathrm{~h}$ para o protocolo Heatsynch. Assim, as inseminações foram realizadas $12 \mathrm{~h}$ antes da ovulação no grupo Ovsynch e oito horas antes da ovulação para o grupo Heatsynch. Ayres et al. ${ }^{22}$ relatam que inseminações realizadas próximo à ovulação apresentam menor taxa de concepção e podem comprometer a eficiência de proto- 
colos de sincronização da ovulação para IATF. Além disso, vacas submetidas ao protocolo Ovsynch apresentam maior taxa de ovulação do que vacas sincronizadas com o protocolo Heatsynch. ${ }^{15}$ Esses resulta$\operatorname{dos}^{15,16,17,18,19,20,21,22}$, indicam que a diferença na taxa de prenhez verificada no presente experimento, a favor das vacas sincronizadas com o protocolo Ovsynch, poderiam ser explicados devido à maior taxa de ovulação e pelo momento mais apropriado para realização da inseminação artificial.

Com o uso do protocolo Heatsynch, existe relato de que as vacas apresentam maior tônus uterino, facilidade em passar a cérvix e sinais de estro ${ }^{10}$, o que pode colaborar para aumentar a utilização da inseminação artificial em tempo fixo por facilitar a realização da técnica (IATF). Esse conjunto de alterações no comportamento e na fisiologia do animal ocorre devido ao efeito positivo do estradiol no comportamento do estro e no aumento do tônus do sistema genital feminino $^{23}$. Porém, esses fatores não foram suficientes para auxiliar no aumento da taxa de prenhez de vacas holandesas submetidas à inseminação artificial em tempo fixo nesse experimento (Tabela 2). Por outro lado, em locais onde as condições das instalações são precárias, a redução dos sinais de estro pode ser uma alternativa para evitar acidentes ${ }^{10}$.

Outro objetivo desse experimento foi avaliar a possibilidade de redução da dose de GnRH no início do protocolo de sincronização da ovulação (Ovsynch e Heatsynch) para IATF. Os resultados apontam que a redução da dose de GnRH no início do protocolo não acarretou redução nos índices reprodutivos em ambos os protocolos (Tabela 3). Dados semelhantes foram obtidos por Fricke, Guenther e Wiltbank ${ }^{8}$, que observaram taxas de concepção aos 28 e 56 dias após a inseminação artificial similares em vacas sincronizadas com 50\% ou $100 \%$ da dose recomendada de GnRH (50 vs $100 \mu \mathrm{g}$ de gonadorelina) no protocolo Ovsynch $(41,0 \%$ vs $41,1 \%)$. Nos protocolos Ovsynch e He- atsynch, o fármaco que apresenta maior custo é o GnRH. A dose inicialmente proposta para o protocolo Ovsynch teve como base a recomendação do fabricante para o tratamento de cisto folicular. No entanto, outros autores ${ }^{24}$ demonstraram que a redução da dose GnRH em 50\% induziu uma resposta de liberação de LH similar a dose total e que ambas as doses foram eficazes no tratamento de cisto foliculares, indicando que a redução da dose de GnRH pode efetivamente induzir ovulação e promover crescimento sincronizado da onda folicular nos protocolos Ovsynch e Heatsynch. Há algum tempo, a utilização dos protocolos para inseminação artificial em tempo fixo (Ovsynch) somente era implantada em fazendas de leite para o tratamento de vacas com presença de cisto folicular. Com a descoberta que esses protocolos poderiam ser utilizados em vacas em um pós-parto recente, inúmeras fazendas passaram a utilizar o protocolo Ovsynch na primeira inseminação artificial do período pósparto por melhorar os índices reprodutivos, comparado aos resultados obtidos com a inseminação artificial seguida da observação de estro, principalmente em vacas de alta produção ${ }^{4}$.

As perdas gestacionais não foram diferentes entre os protocolos (Ovsynch e Heatsynch) e os grupos que receberam $10 \mu \mathrm{g}$ ou $20 \mu \mathrm{g}$ acetato de buserelina no início do protocolo de sincronização (Tabelas 2 e 3). Em vacas de leite, existem relatos de que somente $48 \%$ dos embriões são classificados como normais sete dias após IA $^{25}$. Outros trabalhos ${ }^{26,27}$ indicaram que ocorre uma perda considerável dos embriões antes do exame ultrassonográfico realizado aos 28 dias. Perdas gestacionais de $13,5 \%{ }^{7}$ e $16,8 \%{ }^{28}$ foram verificadas entre 30 e 60 dias após IATF. Valores semelhantes foram encontrados no presente estudo (Tabelas 2 e 3). As possíveis causas para essas perdas gestacionais podem ser estresses lactacional pelo aumento da produção de leite ${ }^{29}$, balanço energético negativo acentuado dessa categoria animal ${ }^{30,31,32}$, efeitos tóxicos da ureia e nitrogênio ${ }^{33}$, estresse térmico 
e a redução da habilidade do animal em responder ao aumento de temperatura do ambiente ${ }^{34}$.

\section{Conclusão}

Com base nesses resultados é possível concluir que o protocolo Ovsynch apresentou maior taxa de prenhez à IATF que o protocolo Heatsynch e que a redução na dose de Acetato de Buserelina no início dos tratamentos de sincronização não comprometeu a eficiência do protocolo.

\section{Agradecimentos}

À Ouro Fino Saúde Animal Ltda pelo financiamento do projeto e à Policlínica Veterinária Pioneiros pelo auxílio técnico e por fornecer os animais utilizados no experimento.

\section{Referências}

1.BARUSELLI, P. S.; AYRES, H.; SOUZA, A. H.; MARTINS, C. M.; GIMENES, L. U.; TORRES JUNIOR, J. R. S. Impacto da IATF na eficiência reprodutiva em bovinos de corte. In: SIMPÓSIO INTERNACIONAL DE REPRODUÇÃO ANIMAL APLICADA, 2., 2006, Londrina: Anais... São Paulo: VRA-FMVZ, 2006. p. 113-132.

2.THATCHER, W. W.; DROST, M.; SAVIO, J. D.; MACMILLAN, K. L.; ENTWISTLE, K. W.; SCHMITT, E. J. P.; DE LA SOTA, R. L. New clinical uses of GnRH and its analogue in cattle. Animal Reproduction Science, v. 33, n. 1/4, p. 27-49, 1993.

3.PURSLEY, J. R.; MEE, M. O.; WILTBANK, M. C. Synchronization of ovulation in dairy cows using PGF2 $\alpha$ and GnRH. Theriogenology, v. 44, n. 7, p. 915-923, 1995.

4.PURSLEY, J. R.; KOSOROK, M. R.; WILTBANK, M. C. Reproductive management of lactating dairy cows using synchronization of ovulation. Journal of Dairy Science, v. 80, n. 2, p. 301-306, 1997.

5.BARUSELLI, P. S.; REIS, E. L.; MARQUES, M. O.; NASSER, L. F. T.; BÓ, G. A. The use of hormonal treatments to improve reproductive performance of anestrous beef cattle in tropical climates. Animal Reproduction Science, v. 82-83, p. 479-486, 2004. Work presented in volumes 82-83, July 2004, Research and Practice III. $15^{\text {th }}$ International Congress of Animal Reproduction.

6.THATCHER, W. W.; MOREIRA, F.; PANCARCIA, S. M.; BARTOLOME, J. A.; SANTOS, J. E. P. Strategies to optimize reproductive efficiency by regulation of ovarian function. Domestic Animal Endocrinology, v. 23, n. 1, p. 243-254, 2002.

7. YANIZ, J. L.; MURUGA, V. E. L. K.; LOPEZ-GATIUS, F. Recent developments in oestrous synchronization of postpartum dairy cows with and without ovarian disorders. Reproduction in Domestic Animal, v. 39, n. 2, p. 86-93, 2004.

8.FRICKE, P. M.; GUENTHER, J. N.; WILTBANK, M. C. Efficacy of decreasing the dose of $\mathrm{GnRH}$ used in a protocol for synchronization of ovulation and timed AI in lactating dairy cows. Theriogenology, v. 50, n. 8, p. 1275-1284, 1998.

9. OLIVEIRA, J. V. L.; RIBEIRO FILHO, A. L. Efeito da dosagem hormonal sobre a fertilidade e a função luteal de vacas zebus sincronizadas com uma combinação de $\mathrm{GnRH}$ e prostaglandina. Revista Brasileira de Reprodução Animal, v. 25, n. 3, p. 323-326, 2001.

10.PANCARCI, S. M.; JORDAN, E. R.; RISCO, C. A.; SCHOUTEN, M. J.; LOPES, F. L.; MOREIRA, F.; THATCHER, W. W. Use of estradiol cypionate in a presynchronized timed artificial insemination program for lactating dairy cattle. Journal of Dairy Science, v. 85, n. 1, p. 122-131, 2002.

11.WILDMAN, E. E.; JONES, G. M.; WAGNER, P. E.; BOMAN, R. L.; TROUTT JR, H. F.; LESCH, T. N. A dairy cow body condition scoring system and its relationship to selected production characteristics. Journal of Dairy Science, v. 65, n. 3, p. 495-501, 1982.

12.NATIONAL RESEARCH COUNCIL (NRC). Nutrient requirements in dairy cattle. Washington: National Academy Press, 2001. 408 p.

13. STATISTICAL ANALYSIS SYSTEM (SAS). SAS user's guide: statistics. North Carolina: SAS Institute Inc, 2001. Disponível em: <http://support.sas.com/documentation/onlinedoc/stat. html>. Acesso em: 17 feb. 2008.

14.KASIMANICKAM, R.; CORNWELL, J. M.; NEBEL, R. L. Fertility following fixed-time AI or insemination at observed estrus in Ovsynch and Heatsynch programs in lactating dairy cows. Theriogenology, v. 63, n. 9, p. 2550-2559, 2005.

15.STEVENSON, J. S.; TIFFANY, S. M.; LUCY, M. C. Use of estradiol cypionate as a substitute for $\mathrm{GnRH}$ in protocols for synchronizing ovulation in dairy cattle. Journal of Dairy Science, v. 87, n. 10, p. 3298-3305, 2004.

16.SAlES, J. N. S.; CREPALDI, G. A.; CARVALHO, J. B. P.; GIROTTO, R. W.; MAIO, J. R. G.; RODRIGUES, M. P.; CARVALHO, C. A. B.; FERREIRA, R. M.; AYRES, H.; BARUSELLI, P. S. Momento da ovulação e taxa de concepção de vacas Nelore tratadas com diferentes fontes de cipionato ou de benzoato de estradiol para induzir a ovulação em protocolos de IATF. In: REUNIÂO ANUAL DA SOCIEDADE BRASILEIRA DE TECNOLOGIA DE EMBRIÕES, 22. 2008, Guarujá. Anais... Rio Grande do Sul: Acta Scientiae Veterinariae, 2008. v. 34, p. 274-2

17. CREPALDI, G. A.; SALES, J. N. S.; MARQUES, M. O.; RIBEIRO JUNIOR, M.; SILVA, R. C. P.; PINHO, J. P. D.; FARIA JUNIOR, S. P.; BARUSELLI, P. S. Rate and timing of ovulation and pregnancy rate in Nelore cows treated with estradiol Cypionate or Benzoate to induce ovulation on FTAI protocols. In: INTERNATIONAL CONGRESS ON ANIMAL REPRODUCTION, 16., 2008, Budapeste. Anais... Berlim: Reproduction in Domestic Animals, 2008. p. 175-175.

18.SAlES, J. N. S.; CARVAlHO, J. B. P.; CREPALDI, G. A.; MAIO, J. R. G.; CARVALHO, C. A. B.; BARUSELLI, P. S. Rate and timing of ovulation in Nelore cows treated with estradiol Cypionate or Benzoate to induce ovulation on FTAI protocols. In: INTERNATIONAL CONGRESS ON ANIMAL REPRODUCTION, 16., 2008, Budapeste. Anais... Berlim: Reproduction in Domestic Animals, 2008. p. 181-181.

19. BARUSELLI, P. S.; MARQUES, M. O.; CARVALHO, N. A. T.; BERBER, R. C. A.; VALENTIM, R.; CARVALHO FILHO, A. 
F.; COSTA NETO, W. P. Follicular dynamics and pregnancy rate in embryo recipient (Bos taurus indicus $\mathrm{x}$ Bos taurus taurus) treated with "Ovsynch" protocol for fixed-time embryo transfer. Brazilian Journal of Veterinary Research and Animal Science, v. 40, p. 96-106, 2003.

20. MARTÍNEZ, M.; KASTELIC, J.; COLAZO, M.; MAPLETOFT, R. Effects of estradiol on gonadotrophin release, estrus and ovulation in CIDR-treated beef cattle. Domestic Animal Endocrinology, v. 33, n. 1, p. 77-90, 2007.

21.MAIO, J. R. G.; SANDOVAL, G. A. F.; SOUZA, E. D. F.; NOGUEIRA, G. P.; CIPRIANO, R. S.; PERECIN, F.; GARCIA, J. M. Concentração sérica de LH em vacas Nelore ciclando ou ovariectomizadas submetidas ao tratamento com 2,0mg de Benzoato de estradiol. In: REUNIÃO ANUAL DA SOCIEDADE BRASILEIRA DE TECNOLOGIA DE EMBRIÕES, 20., 2006, Araxá. Anais... Rio Grande do Sul: Acta Scientiae Veterinariae, 2006. v. 32, p. 360-360.

22.AYRES, H.; MARTINS, C. M.; FERREIRA, R. M.; MELLO, J. E.; DOMINGUEZ, J. H.; SOUZA, A. H.; VALENTIN, R.; SANTOS, I. C. C.; BARUSELLI, P. S. Effect of timing of estradiol benzoate administration upon synchronization of ovulation in suckling Nelore cows (Bos indicus) treated with a progesterone-releasing intravaginal device. Animal Reproduction Science, v. 109, n. 1/4, p. 77-87, 2008.

23. PERRY, G. A.; PERRY, B. L. Effect of preovulatory concentrations of estradiol and initiation of standing estrus on uterine $\mathrm{pH}$ in beef cows. Domestic Animal Endocrinology, v. 34, n. 3, p. 333-338, 2008.

24.SEGUIN, B. E.; CONVEY, E. M.; OXENDER, W. D. Effect of gonadotropin-releasing hormone and human chorionic gonadotropin on cows with ovarian follicular cysts. American Journal of Veterinary Research, v. 37, n. 2, p. 153-157, 1976.

25.WEIBOLD, J. L. Embryonic mortality and the uterine environment in first-service lactating dairy cows. Journal of Reproduction and Fertility, v. 84, n. 2, p. 393-395, 1988.
26.KUMMERFELD, H. L.; OLTENACU, E. A. B.; FOOTE, R. H. Embryonic mortality in dairy cows estimated by nonreturns to service, estrus, and cyclic milk progesterone patterns. Journal of Dairy Science, v. 61, n. 12, p. 1773-1777, 1978.

27.SARTORI, R.; GÜMEN, A.; GUENTHER, J.; SOUZA, A.; CARAVIELLO, D.; WILTBANK, M. Comparison of artificial insemination versus embryo transfer in lactating dairy cows. Theriogenology, v. 65, n. 7, p. 1311-1321, 2006.

28.VASCONCELOS, J. L. M.; SILCOX, R. W:; ROSA, G. J. M.; PURSLEY, J. R.; WILTBANK, M. C. Synchronization rate, size of the ovulatory follicle, and pregnancy rate after synchronization of ovulation beginning on different days of the estrous cycle in lactating dairy cows. Theriogenology, v. 52, n. 6, p. 1067-1078, 1999.

29. NEBEL, R. L.; MCGILLIARD, M. L. Interactions of high milk yield and reproductive performance in dairy cows. Journal of Dairy Science, v. 76, n. 10, p. 3257-3268, 1993.

30. RHODES, F. M.; MCDOUGALL, S.; BURKE, C. R.; VERKERK, G. A.; MACMILLAN, K. L. Invited Review: Treatment of Cows with an Extended Postpartum Anestrous Interval. Journal of Dairy Science, v. 86, n. 6, p.1876-1894, 2003.

31.RHOADS, M. L.; RHOADS, R. P.; GILBERT, R. O.; TOOLE, R.; BUTLER, W. R. Detrimental effects of high plasma urea nitrogen levels on viability of embryos from lactating dairy cows. Animal Reproduction Science, v. 91, n. 1/2, p. 1-10, 2006.

32.BUTLER, W. I. L.; SMITH, R. D. Interrelationships between energy balance and postpartum reproductive function in diary cattle. Journal of Dairy Science, v. 72, n. 3, p. 767-783, 1989.

33.SANTOS, J. E. P.; CERRI, R. L. A.; SARTORI, R. Nutritional management of the donor cow, Theriogenology, v. 69, n. 8, p. 88-97, 2008.

34.STEVENSON, J. S.; SCHMIDT, M. K.; CALL, E. P. Stage of estrous cycle, time if insemination, and seasonal effects on estrus and fertility of Holstein heifers after prostaglandin F2a. Journal of Dairy Science, v. 67, p. 1798-1805, 1984. 